\title{
Contribution of PET/CT imaging to differential diagnosis of fever of unknown origin
}

\author{
Halcin A, Kinova S \\ 1st Department of Internal Medicine, Comenius University, Bratislava, Slovakia. halcin@zoznam.sk
}

\begin{abstract}
Background: Vasculitis is a collective title for a heterogeneous group of diseases with common signs of inflammation, leukocytic infiltration and necrosis of the vessel wall leading to regional perfusion disturbances. There are many ways to classify vasculitis. Into the group of large-vessel vasculitis we include Takayasu's arteritis as well as temporal arteritis (giant-cell arteritis) affecting also the aorta and its major branches.

Methods: FDG PET/CT is a hybrid imaging method combining spatial imaging of metabolic activity obtained by the detection of 18-fluorodeoxyglucose (FDG) with positron emission tomography (PET) and X-ray computed tomography (CT). While carried out together with PET imaging in the same session, CT imaging is helpful in identifying precisely the anatomical identification of hypermetabolic lesions detected via PET.

Results: In this case report we refer to the key contribution of PET/CT imaging to concluding successfully a diagnostic process lasting for a few months and leading to a revelation of large-vessel vasculitis manifesting itself only with systemic inflammation symptoms, i.e. without any clinical signs of specific organ damage.

Conclusion: In conclusion, FDG PET/CT scan is a combined imaging technique which has a remarkable potential in the diagnosis of large-vessel vasculitis. This potential is particularly valued in cases when symptoms of vasculitis are clinically nonspecific and when other non-invasive methods are failing (Fig. 2, Ref. 20). Full Text in PDF www.elis.sk.

Key words: vasculitis, FDG, PET/CT, fever of unknown origin.
\end{abstract}

Vasculitis is a collective title for a heterogeneous group of diseases with common signs of inflammation, leukocytic infiltration, and necrosis of the vessel wall, leading to regional perfusion disturbances. According to type of organ affected with ischemia, specific clinical correlates may be present. In almost all cases we can find also nonspecific symptoms generally seen in many other diseases, such as fever, nocturnal sweating, weakness, loss of weight, and arthralgia. Highly elevated erythrocyte sedimentation rate and C-reactive protein, as well as changes in blood count (leucocytosis, thrombocytosis, anaemia) are frequent findings in results of laboratory examinations.

There are many ways of classifying vasculitis. One of them, classification from Chapel Hill (1993), classifies vasculitis by caliber of the vessel predominantly affected. Accordingly, we can discern vasculitis of large, medium and small vessels (1).

Into the group of large-vessel vasculitis we include Takayasu's arteritis, as well as temporal arteritis (giant-cell arteritis) affecting also the aorta and its major branches. These two diseases affect different groups of patients. Takayasu's arteritis typically affects

1st Department of Internal Medicine, Comenius University, Bratislava, Slovakia

Address for correspondence: A. Halcin, MD, Ambroseho 3, SK-851 02, Bratislava, Slovakia.

Phone: +421.949733197, Fax: +421.2 .57290673$

Acknowledgements: The authors would like to thank Dr. Emoke Šteňová and Dr. Pavol Povinec for their treasured help. The authors have no financial or other conflicts of interest. women younger than 40 years, whereas giant-cell arteritis usually occurs in older patients, typically over 50-60 years, often accompanied with symptoms of polymyalgia rheumatica (2).

The non-invasive diagnostic method of 18-fluorodeoxyglucose (FDG) positron emission tomography (PET) with computed tomography (FDG PET/CT) is relatively new. It is a hybrid imaging method combining spatial imaging of metabolic activity obtained by the detection of 18-fluorodeoxyglucose done with positron emission tomography and an X-ray computed tomography (CT). $\mathrm{CT}$ imaging is helpful in providing a more precise anatomical identification of areas with accumulated FDG detected via PET in the same session.

FDG after being transported into a cell is phosphorylated to 2-FDG-6-phosphate which is a molecule that cannot leave the cell and thus accumulates in it.

High accumulation of FDG is typically seen in most types of malignant cells but also in some types of inflammatory cells. So beside more frequent oncological diagnoses, FDG PET is often also used to localise inflammatory processes.

First publications illustrating the ability of FDG PET examination to reveal the presence of large-vessel vasculitis appear in literature in 1999. In this year, Blockmans et al. published case reports concerning FDG PET detection of giant-cell arteritis and polymyalgia rheumatica. In the same year, Hara et al. described FDG PET detection in Takayasu's arteritis $(3,4)$.

Recently, the value of this examination started to be studied in a wider range of chronic inflammatory diseases such as retroperi- 
toneal fibrosis (5), systemic sarcoidosis (6), rheumatoid arthritis $(7)$, spondyloarthritis $(8,9)$ etc., in order to assess the disease activity, or in monitoring the response to treatment.

In this case report we refer to the key contribution of FDG PET/ CT imaging to concluding successfully a diagnostic process lasting for a period of few months and leading to a revelation of largevessel vasculitis manifesting itself only with systemic inflammatory symptoms, i.e. without specific clinical signs of organ lesions.

\section{Case report}

A 57-year-old female with history of thyroidal hypofunction and postmenopausal osteoporosis started to suffer from a painful swelling in the left submandibular region, accompanied with subfebrile states. The troubles were assessed as tonsillitis. After an antibiotic treatment, the swelling disappeared but in the next period, a long-term febrile state, nocturnal sweating, anorexia with loss of weight, arthralgia and exhaustion appeared.

Laboratory tests revealed microcytic anaemia, leucocytosis, thrombocytosis and highly elevated markers of inflammation (ESR $>100 / \mathrm{h}$, CRP $102 \mathrm{mg} / \mathrm{l}$ ). Despite these laboratory signs of inflammation, plasmatic level of procalcitonin was in normal range. Because of serological detection of Mycoplasma pneumoniae infection, the patient was treated with corresponding antibiotic therapy but no significant changes of overall condition were witnessed. Microbiological (urine, nasal and tonsil swabs, bronchoalveolar lavage, haemocultures) and serological examinations (hepatitis B, syphilis, HIV, cytomegalovirus, Epstein-Barr virus) were not able to detect any other pathogen. Gynaecological and otorhinolaryngological examinations were also negative. Endocrinologic as well as ultrasonographic examinations excluded the possibility of subacute thyreoiditis.

After repeated antibiotic therapy (ciprofloxacin, azithromycin, doxycyclin, clarithromycin) without any significant effect, a suspicion of an oncological process was pronounced. The patient underwent a wide range of laboratory and imaging examinations but with no positive findings. CT scan of thorax, abdomen and pelvis showed a small pericardial effusion and increased number of lymphatic nodes in anterior mediastinum with maximal size of 14 millimetres in subcarinal area, without any other pathologic structural finding. Trepanobiopsy of bone marrow and subsequent haematological examinations were not helpful, just as well as the search for tuberculosis (negative results of PPD skin test and Quantiferon test). Echocardiography showed normal cardiac morphology and function with a little pericardial effusion. CT scan of head and neck revealed an increased number of lymphatic nodes up to a size of 10 millimetres fusing to packets in the central thirds of both sides of the neck.

FDG PET/CT scan was performed where the nodosity of thyroidal gland was reported however without the confirmation of metabolically active lymphadenopathy in the cervical area. Nevertheless, a diffusely increased metabolism of 18-FDG was found in the vessel wall of the thoracic and abdominal parts of aorta, common iliac, subclavian and axillar arteries, as well as in the vessel wall of both common carotid arteries. This finding was

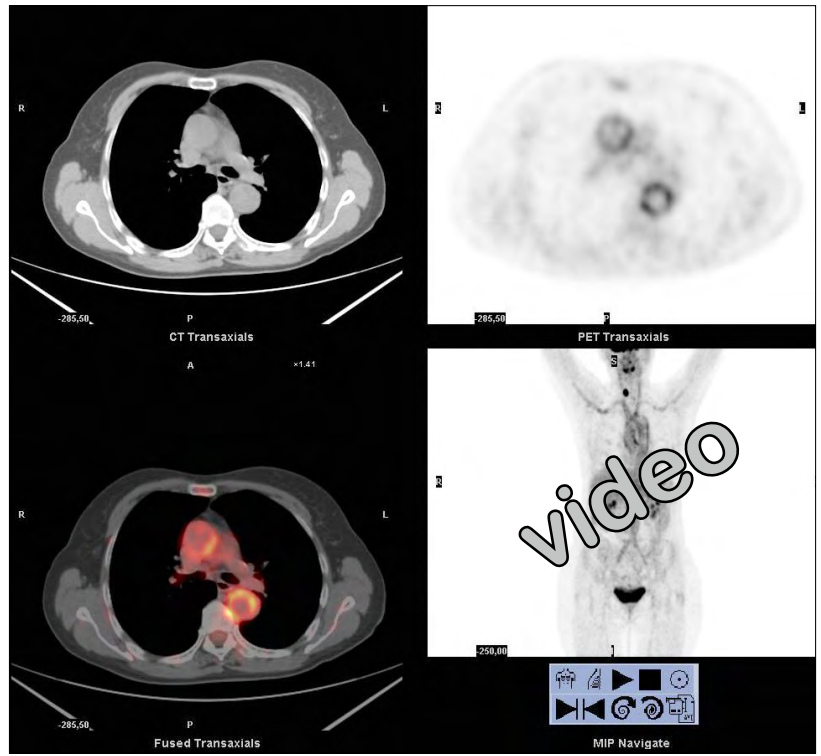

Fig. 1. FDG-PET/CT image of a patient with vasculitis. Clock-wise starting from top left: axial CT, axial PET, PET maximum intensity projection, fused PET/CT image. Note circumferentially increased FDG uptake in walls of large vessels, including thoracic and abdominal aorta, subclavian, axillar, common carotid and common iliac arteries. Accidental finding of a focal uptake in the right lobe of the thyroid gland, which was later confirmed to be a benign adenoma.
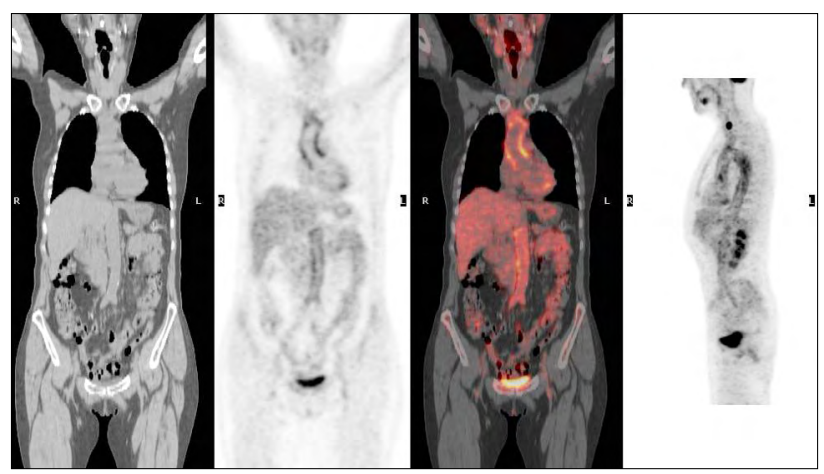

Fig. 2. FDG-PET/CT image. Starting from left: coronal CT, coronal PET, fused PET/CT image and PET maximum intensity projection,. Increased FDG uptake is in these slices best marked in walls of thoracic and abdominal aorta, In fourth projection, besides very well seen aorta, also big branches of aortal arch and common iliac arteries are well marked.

assessed as an evident manifestation of highly active large-vessel vasculitis (Fig. 1).

Ir is well known from literature that the 18- FDG PET pictures of cases with rheumatic polymyalgia, Takayasu's arteritis and giant-cell arteritis are very similar. Hypermetabolism of 18- FDG is in all these cases usually found in the vessel wall of the aortic arch, ascendent, descendent and abdominal parts of aorta as well as in their main branches. Thus in all these cases, the conclusion of FDG PET finding usually refers to "large-vessel vasculitis" or "largevessel arteritis", without any further differentiation $(10,11,12)$. 
This means that the differential diagnosis between these nosological units must be usually done in other ways, especially in clinical manner. Considering the age and clinical symptoms of our patient, in this case we conclude the presence of giant-cell arteritis.

Prednisone in an overall dosage of 50 milligrams per day, in combination with $100 \mathrm{mg}$ of azathioprin per day was prescribed. After one month of this therapy, our patient witnessed a significant subjective improvement of her health conditions.

The blood count and the levels of inflammatory markers have also fully normalised. The presence of autoimmune vasculitis was approved also by a therapeutic test with immunosuppressive doses of corticotherapy.

\section{Discussion}

Our article describes a case of a long-term and wide-range diagnostic process in a patient with fever of unknown origin persisting for several months. The initial search for infectious disease was replaced with an effort to reveal a neoplasm. Thereafter we progressed to a diagnosis of autoimmune inflammation. Largevessel vasculitis was in this case clinically manifesting itself only with nonspecific symptoms of systemic inflammation, i.e. without any presence of significant organ changes that could have possibly lead us directly to the diagnostic solution or enabled us to take bioptic samples.

After achieving a list of negative results from more elementary and available examinations, a FDG PET/CT scan has been shown to be the crucial step in the diagnostic process because it displayed a distribution of inflammatory changes typical for a large-vessel vasculitis.

Large-vessel vasculitis is considered to be the cause of fever of unknown origin in $17 \%$ of all patients suffering from the latter condition (FUO) (13).

PET is a nuclear medicine imaging technique which produces a three-dimensional image of metabolic processes in the body. When the biologically active molecule used for this examination is ${ }^{18} \mathrm{FDG}$, an analogue of glucose, the resultant image shows tissue metabolic activity in terms of regional glucose uptake. Due to increased regional glucose uptake, ${ }^{18} \mathrm{~F}$-FDG PET is able to show distribution of processes with high metabolic activity such as most types of malignant tumours, as well as an inflammatory tissue under some specific conditions.

The percentage of PET scans helpful in the diagnostic process in patients with FUO varies according to literature from 41 to $69 \%$ (14).

In case of large-vessel vasculitis, FDG-PET is highly effective in detecting it anywhere in the body with a high corresponding sensitivity (77-100\%) and specificity (89-100\%) (15).

Under authority of literal sources, in cases of large vessel vasculitis, a correlation between metabolic activity detected by FDG PET scan and elevated levels of inflammatory parameters (CRP, ESR), platelets and with a level of haemoglobin in inverse way have been proved $(11,16,17,18)$. This is also a piece of information witnessing the diagnostic possibilities of FDG PET scan in this area.
FDG PET scans are also being used to follow up patients over time as well as to monitor the response to anti-inflammatory and immunosuppressive treatment. Following literal sources, control FDG PET scans carried out after the administration of corticosteroids in patients with large-vessel vasculitis showed a remarkable decrease as well as disappearance of metabolic activity of disease that correlated with changes in clinical and laboratory parameters of disease activity

(16-19).

In conclusion, FDG PET/CT scan is a combined imaging technique which has a remarkable potential in the diagnosis of largevessel vasculitis. This potential is particularly valued in cases with symptoms of vasculitis that is clinically nonspecific and manifests itself only with symptoms of fever of unknown origin or when other non-invasive methods are failing.

In addition to being able to state the diagnosis, FDG PET/CT scan provides valuable information about activity, extent and localisation of the inflammatory process. In accordance with literature, FDG PET/CT scan was able to assess the diagnosis of largevessel vasculitis also in many cases, in which $\mathrm{CT}$ angiography or MR angiography had failed (4, 18, 20).

This takes place in the early stage of the disease when the typical structural vessel wall changes are not yet developed to an extent to be able to condition the angiographic image of the disease.

Some limitation of FDG PET scan lies in its inability to assess arteries with a diameter of less than 4-5 millimetres. The diagnostic approach with FDG PET scan is rational but only before the ordination of immunosuppressive therapy which suppresses the metabolic activity in the vessel wall that ought to be visualised.

\section{Learning points}

As shown in this case, also the very extensive autoimmune process of vasculitis can manifest itself with systemic inflammatory symptoms only, i.e. without any organ-specific pathognomic signs.

Large-vessel vasculitis is considered to be the cause in $17 \%$ of all FUO patients.

High sensitivity and specificity of FDG-PET is highly effective in detecting the large-vessel vasculitis.

FDG PET scan can also be used to follow up patients over time and to monitor the activity of autoimmune vessel-wall process as well as its response to anti-inflammatory and immunosuppressive treatment.

\section{References}

1. Jennette JC, Falk RJ, Andrassy K et al. Nomenclature of systemic vasculitides: proposal of an international consensus conference. Arthritis Rheum 1994; 37: 187-192.

2. Pavelka K. Polymyalgia rheumatica a temporální arteriitida: přehledný referát. Čes Revmatol 2001; 3: 129-136.

3. Blockmans D, Maes A, Stroobants $\mathbf{S}$ et al. New arguments for a vasculitic nature of polymyalgia rheumatica using positron emission tomography. Rheumatology (Oxford) 1999; 38: 444-447. 
4. Hara M, Goodman PC, Leder RA. FDG-PET finding in early-phase Takayasu arteritis. J Comput Assist Tomogr 1999; 23: 16-18.

5. Jansen I, Hendriksz TR, Han SH, Huiskes AW, van Bommel EF. (18)F-fluorodeoxyglucose position emission tomography (FDG-PET) for monitoring disease activity and treatment response in idiopathic retroperitoneal fibrosis. Eur J Intern Med 2010; 21 (3): 216-221.

6. Desvignes A, Zuily S, Saadi L, Olivier P, Regent D, Schuhmacher H, Wahl D. FDG-PET to monitor early response to infliximab in refractory systemic sarcoidosis. Respiratory Medicine Extra. 2007; 3 (4): 178-180.

7. Dos Anjos DA, do Vale GF, de Mello CC, et al. Extra-Articular Inflammatory Sites Detected by F-18 FDG PET/CT in a Patient With Rheumatoid Arthritis. Clin Nucl Med 2010; 35 (7): 540-541.

8. Taniguchi Y, Arii K, Kumon Y, Fukumoto M et al. Positron emission tomography/computed tomography: a clinical tool for evaluation of enthesitis in patients with spondyloarthritides. Rheumatology 2010; 49 (2): 348-354.

9. Wendling D, Blagosklonov O, Streit G, Lehuédé G, Toussirot E, Cardot J-C. FDG-PET/CT scan of inflammatory spondylodiscitis lesions in ankylosing spondylitis, and short term evolution during anti-tumour necrosis factor treatment. Ann Rheum Dis 2005; 64: 1663-1665.

10. Wenger M, Gasser R, Donnemiller E et al. Generalized large vessel arteritis visualised by 18 Fluoroglucose-positron emission tomography. Circulation 2003; 107: 923.

11. Walter MA, Melzer RA, Schindler CH, Müller-Brand J, Tyndall A, Nitzsche EU. The value of [18F]FDG PET in the diagnosis of large vessel vasculitis and the assessment of activity and extent of disease. Eur J Nucl Med Mol Imaging 2005; 32: 674-681.

12. Wiest R, Glück T, Schönberger J, Schölmerich J, Eilles C, MüllerLadner U. Clinical image: occult large vessel vasculitis diagnosed by PET imaging. Rheumatol Int 2001; 20: 250.
13. Vanderschueren S, Knockaert D, Adriaenssens T, Demey W, Durnez A, Blockmans D, Bobbaers H. From prolonged febrile illness to fever of unknown origin: the challenge continues. Arch Intern Med 2003; 163 (9): 1033-1041.

14. Bleeker-Rovers CP, de Kleijn EM, Corstens FH, Meer JW, Oyen WJ. Clinical value of FDG PET in patients with fever of unknown origin and patients suspected of focal infection and inflammation. Eur J Nucl Med Mol Imaging 2004; 31: 29-37.

15. Otsuka H, Morita N, Yamashita K, Nishitani H. FDG-PET/CT for diagnosis and follow up of vasculitis J Med Inv 2007; 54: 345-349.

16. Moosig F, Czech N, Mehl C, Henze E, Zeuner RA, Kneba M, Schroder JO. Correlation between 18-fluorodeoxyglucose accumulation in large vessels and serological markers of inflammation in polymyalgia rheumatica: a quantitative PET study. Ann Rheum Dis 2004; 63: 870-873.

17. Scheel AK, Meller J, Vosshenrich R, Kohlhoff E, Siefker U, Müller GA, Strutz F. Diagnosis and follow up of aortitis in the elderly. Ann Rheum Dis 2004; 63: 1507-1510.

18. Řehák Z, Fojtík Z, Staníček J, Bolčák K, Fryšáková L. 18FFDG PET v diagnostice vaskulitid velkých cév. Vnitř Lék 2006; 52 (11): 1002-1003.

19. Šteňová E, Mištec S, Povinec P. FDG-PET/CT in large- vessel vasculitis: its diagnostic and follow-up role. Rheumatol Int 2010; 30 (8): 1111-1114.

20. Meller J, Grabe E, Becker W, Vosshenrich R. Value of 18-FDG hybrid camera PET and MRI in early Takayasu aortitis. Eur Radiol 2003; 13: $400-405$.

Received February 3, 2011. Accepted August 18, 2012. 